\title{
Bounded circular distortion curves and quasidisks*
}

\author{
Yuming ChU and Zhenjiang ZHAO
}

(Received December 24, 2002; Revised May 12, 2003)

\begin{abstract}
Let $D$ be a Jordan domain in $\bar{R}^{2}$ and $\Gamma=\partial D$ be the boundary of $D$. Then $D$ is a quasidisk if and only if $\Gamma$ is a bounded circular distortion curve.

Key words: bounded circular distortion curve, quasidisk, quasiconformal, mapping.
\end{abstract}

\section{Introduction}

Let $D$ be a Jordan domain in $\bar{R}^{2}$ and $f: \bar{R}^{2} \rightarrow \bar{R}^{2}$ be a $k$-quasiconformal mapping, where $1 \leq k<+\infty$. $D$ is called a quasidisk if $D$ is the image of the unit disk $B^{2}$ under $f$.

It is well-known that quasidisks play a very important role in quasiconformal mapping theory, complex dynamics, Fuchsian groups, Teichmüller space theory and low dimensional topology, see $[2,3,7,9,11]$ etc.

In 1963, L.V. Ahlfors obtained the three-point property of quasidisks ([1]). Later, F.W. Gehring [5], B.G. Osgood [10], J.G. Krzyz [8], Y. Chu and J. Cheng [4] studied the quasidisks extensively. Several characterizations of quasidisks were obtained. In this paper, we shall prove a new characterization of quasidisks.

Definition 1 Let $E$ be a set in $\bar{R}^{2}$ and $c \geq 1$ be a constant. $E$ is called a $c$-linearly locally connected set if for any $x \in R^{2}$ and $0<r<+\infty$, the following are satisfied:

(1) any two points in $E \cap \bar{B}^{2}(x, r)$ can be joined by a curve in $E \cap \bar{B}^{2}(x, c r)$; (2) any two points in $E \backslash B^{2}(x, r)$ can be joined by a curve in $E \backslash B^{2}(x, r / c)$.

$E$ is called a linearly locally connected set if $E$ is a $c$-linearly locally connected set for some $c \geq 1$.

F.W. Gehring and O. Martio obtained the following result ([6]):

2000 Mathematics Subject Classification : 30C62.

${ }^{*}$ The research in partly supported by N.S. Foundation of China under grant 10471039 and 10271043, N.S. Foundation of Zhejiang province under grant M103087. 
Theorem A If $D$ is a Jordan domain in $\bar{R}^{2}$, then $D$ is a quasidisk if and only if $D$ is a linearly locally connected domain.

Definition 2 A curve $\Gamma \subset \bar{R}^{2}$ is said to be of $c$-bounded circular distortion, where $0<c \leq 1$, if for all $x \in \Gamma \cap R^{2}$ and $r>0$, the disk $B^{2}(x, c r)$ meets only the $x$-component of $\Gamma \cap \bar{B}^{2}(x, r)$. $\Gamma$ is called a bounded circular distortion curve if $\Gamma$ is a $c$-bounded circular distortion curve for some $c$, where $0<c \leq 1$.

Remark 1 It follows from definitions 1 and 2 that a Jordan curve with $c$-bounded circular distortion is $b$-linearly locally connected, where $b=1 / c$.

The main aim of this paper is to prove the following result:

Theorem 1.1 Let $D$ be a Jordan domain in $\bar{R}^{2}$ and $\Gamma=\partial D$ be the boundary of $D$. Then $D$ is a quasidisk if and only if $\Gamma$ is a bounded circular distortion curve.

\section{The proof of Theorem $\mathbf{1 . 1}$}

Lemma 2.1 Let $\Gamma$ be a Jordan curve. If $\Gamma$ is a bounded circular distortion curve, then $\Gamma$ is a quasicircle.

Proof. Since $\Gamma$ is a bounded circular distorsion curve, by definition 2, there exists a constant $c(0<c \leq 1)$ such that $\Gamma$ is a $c$-bounded circular distortion curve.

Suppose first that $\infty \in \Gamma$. Let $x_{1}, x_{2}, x_{3}$ be three points on $\Gamma$ in this order. If $c\left|x_{1}-x_{2}\right|>\left|x_{1}-x_{3}\right|$, then obviously $\Gamma$ is not a $c$-bounded circular distortion curve. Consequently,

$$
\frac{\left|x_{1}-x_{2}\right|}{\left|x_{1}-x_{3}\right|} \leq \frac{1}{c}
$$

It follows from [1, Theorem1] that $\Gamma$ is a quasicircle.

Then suppose that $\Gamma \in R^{2}$. Without loss of generality, we may assume $0<c \leq 1 / 2$. By $\left[1, P_{295}\right], \Gamma$ is a quasicircle if

$$
\frac{\left|x_{1}-x_{2}\right|\left|x_{3}-x_{4}\right|}{\left|x_{1}-x_{3}\right|\left|x_{2}-x_{4}\right|} \leq b
$$

where $x_{i} \in \Gamma(i=1,2,3,4), x_{2}$ and $x_{4}$ lie in different components of $\Gamma \backslash\left\{x_{1}, x_{3},\right\}$. In the following we shall show that (2.2) holds for $b=c^{-4}$. 
Let $x_{i}(i=1,2,3,4)$ be the above stated four points on $\Gamma$, and let $u=$ $\left|x_{1}-x_{2}\right| /\left|x_{1}-x_{3}\right|$. Suppose $u>1 / c^{2}$. Now $\left|x_{1}-x_{4}\right| \leq\left|x_{1}-x_{3}\right| / c$ since otherwise $\Gamma$ is not a $c$-bounded circular distortion curve. It follows that

$$
\left|x_{1}-x_{4}\right| \leq \frac{\left|x_{1}-x_{3}\right|}{c}=\frac{\left|x_{1}-x_{2}\right|}{c u}<c\left|x_{1}-x_{2}\right| .
$$

On the other hand, if we set $a=\left|x_{1}-x_{2}\right| /\left|x_{2}-x_{4}\right|$, then (2.3) implies that

$$
a \leq \frac{\left|x_{1}-x_{4}\right|+\left|x_{2}-x_{4}\right|}{\left|x_{2}-x_{4}\right|} \leq \frac{c\left|x_{1}-x_{2}\right|+\left|x_{2}-x_{4}\right|}{\left|x_{2}-x_{4}\right|}=a c+1 .
$$

Obviously $a \leq 1 /(1-c) \leq 2$. Combining the following inequalities:

$$
\left|x_{3}-x_{4}\right| \leq\left|x_{3}-x_{1}\right|+\left|x_{1}-x_{4}\right| \leq\left(1+\frac{1}{c}\right)\left|x_{1}-x_{3}\right| \leq \frac{2}{c}\left|x_{1}-x_{3}\right|,
$$

we conclude that (2.2) holds with $b=4 / c \leq 1 / c^{4}$.

The cases where $v=\left|x_{3}-x_{4}\right| /\left|x_{2}-x_{4}\right|>1 / c^{2}$ and $u, v \leq 1 / c^{2}$ can be proved in analogous way. These complete the proof.

Remark 2 The result that a Jordan domain $D \subset \bar{R}^{2}$ is a quasidisk if and only if $\partial D$ is linearly locally connected had been proved by M.F. Walker in [12, Corollary 4.4], but the method in the proof of Lemma 2.1 is different from that in [12].

Lemma 2.2 Let $D$ be a Jordan domain and $\Gamma=\partial D$ be the boundary of $D$. If $D$ is a quasidisk, then $\Gamma$ is a bounded circular distortion curve.

Proof. Since $D$ is a quasidisk, by Theorem A, $D$ is a linearly locally connected domain. Then there exists a constant $c \geq 1$ such that $D$ is a $c$-linearly locally connected domain. In the following we shall prove that $\Gamma=\partial D$ is a $1 / c$-bounded circular distortion curve.

Suppose that $\Gamma$ is not a $1 / c$-bounded circular distortion curve. Then there exist $x \in \Gamma \cap R^{2}$ and $r(0<r<+\infty)$ such that $B^{2}(x, r / c)$ meets a component $E_{1}$ of $\Gamma \cap \bar{B}^{2}(x, r)$, which isn't the $x$-component $E_{2}$ of $\Gamma \cap \bar{B}^{2}(x, r)$. Let $G_{i}$ be the component of $B^{2}(x, r) \cap D$ which contains $E_{i}$ as a part of a boundary $(i=1,2)$. There are two possibilities:

(1) $G_{1}=G_{2}$. It is easy to see that there exist points $x_{1}, x_{2} \in D \backslash B^{2}(x, r)$ which can be joined by a curve in $D$ only through $B^{2}(x, r / c)$. Hence $x_{1}$, $x_{2}$ cannot be joined by a curve in $D \backslash B^{2}(x, r / c)$. 
(2) $G_{1} \neq G_{2}$. Choose points $x_{i} \in B^{2}(x, r / c) \cap G_{i}(i=1,2)$. If $x_{1}$ and $x_{2}$ can be joined by a curve $\alpha$ in $D$, then $\alpha$ will meet $\bar{R}^{2} \backslash \bar{B}^{2}(x, r)$. Hence $x_{1}, x_{2}$ cannot be joined by a curve in $D \cap \bar{B}^{2}(x, r)$.

The above shows that $D$ isn't a $c$-linearly locally connected domain. This is a contradiction. Hence $\Gamma$ is a bounded circular distortion curve.

Proof of Theorem 1.1. If $D$ is a quasidisk, then $\Gamma=\partial D$ is a bounded circular distortion curve by Lemma 2.2. On the other hand, if $\Gamma=\partial D$ is a bounded circular distortion curve, then $\Gamma$ is a quasicircle by Lemma 2.1, hence $D$ is a quasidisk.

Acknowledgements This work was completed during the first author's visit to the University of Helsinki. He wishes to express his thanks to the Department of Mathematics of the University of Helsinki for hospitality.

\section{References}

[1] Ahlfors L.V., Quasiconformal reflection. Acta Math. 109 (1963), 291-301.

[2] Balogh Z., Hausdorff dimension distribution of quasiconformal mappings on the Heisenberg group. J. d'Analyse Math. 83 (2001), 289-312.

[ 3 ] Beardon A.F., The geometry of discrete groups. Springer-Verlag, 1982.

[4] Chu Y.M. and Chen J.F., Quasidisks and module monotone domains. Acta Math. Sinica (in Chinese) 39 (1996), 556-560.

[5] Gehring F.W., Uniform domain and the uniquitous quasidisk. Jber. d. Dt. Math. 89 (1987), 88-103.

[6] Gehring F.W. and Martio O., Quasiextremal distance domains and extension of quasiconformal mappings. J. Analyse Math., 45 (1985), 181-206.

[7] Koranyi A. and Reimann H.M., Foundations for the theory of quasiconformal mappings on the Heisenberg group. Adv. In Math. 111 (1995), 1-87.

[8] Krzyz J.G., Quasicircles and harmonic measure. Ann. Acad. Sci. Fenn. Math. 12 (1987), 19-24.

[9] Lehto O., Univalent functions and Teichmüller space. Springer-Verlag, 1986.

[10] Osgood B.G., Univalence criteria in multiply-connected domains. Trans. Amer. Math. Soc. 260 (1980), 499-519.

[11] Sullivan D., Quasiconformal homeomorphisms and dynamics II. Acta Math. 155 (1985), 243-260.

[12] Walker M.F., Linearly locally connected sets and quasiconformal mappings. Ann. Acad. Sci. Fenn. Math. 11 (1986), 77-86. 
Y. Chu

Department of Mathematics

Huzhou Normal college

Huzhou, Zhejiang 313000

P. R. China

E-mail: chuyuming@hutc.zj.cn

Z. Zhao

Department of Mathematics

Huzhou Normal college

Huzhou, Zhejiang 313000

P. R. China

E-mail: zhaozjcn@163.com 